\title{
Cospectral graphs and regular orthogonal matrices of level 2
}

\author{
Aida Abiad* Willem H. Haemers \\ Department of Econometrics and Operations Research \\ Tilburg University, Tilburg, The Netherlands \\ aidaabiad@gmail.com haemers@uvt.nl
}

Submitted: May 24, 2012; Accepted: Jul 25, 2012; Published: Aug 9, 2012

Mathematics Subject Classifications: 05B20

\begin{abstract}
For a graph $\Gamma$ with adjacency matrix $A$, we consider a switching operation that takes $\Gamma$ into a graph $\Gamma^{\prime}$ with adjacency matrix $A^{\prime}$, defined by $A^{\prime}=Q^{\top} A Q$, where $Q$ is a regular orthogonal matrix of level 2 (that is, $Q^{\top} Q=I, Q \mathbf{1}=\mathbf{1}, 2 Q$ is integral, and $Q$ is not a permutation matrix). If such an operation exists, and $\Gamma$ is nonisomorphic with $\Gamma^{\prime}$, then we say that $\Gamma^{\prime}$ is semi-isomorphic with $\Gamma$. Semiisomorphic graphs are $\mathbb{R}$-cospectral, which means that they are cospectral and so are their complements. Wang and $\mathrm{Xu}$ ['On the asymptotic behavior of graphs determined by their generalized spectra', Discrete Math. 310 (2010)] expect that almost all pairs of nonisomorphic $\mathbb{R}$-cospectral graphs are semi-isomorphic.

Regular orthogonal matrices of level 2 have been classified. By use of this classification we work out the requirements for this switching operation to work in case $Q$ has one nontrivial indecomposable block of size 4,6,7 or 8. Size 4 corresponds to Godsil-McKay switching. The other cases provide new methods for constructions of $\mathbb{R}$-cospectral graphs. For graphs with eight vertices all these constructions are carried out. As a result we find that, out of the 1166 graphs on eight vertices which are $\mathbb{R}$-cospectral to another graph, only 44 are not semi-isomorphic to another graph.
\end{abstract}

\section{Introduction}

An orthogonal matrix $Q$ is regular if it has constant row sum, that is, $Q \mathbf{1}=r \mathbf{1}$ (where $\mathbf{1}$ is the all-one vector). From $Q^{\top} Q=Q Q^{\top}=I$, it follows that also $Q^{\top} \mathbf{1}=r \mathbf{1}$, and that $r= \pm 1$. Without loss of generality we will assume $r=1$. A regular orthogonal matrix has level $\ell$ if $\ell$ is the smallest positive integer such that $\ell Q$ is an integral matrix. We define $\ell=\infty$ if $Q$ has irrational entries. Clearly $\ell=1$ if and only if $Q$ is a permutation matrix.

\footnotetext{
*supported by the Netherlands Organisation for Scientific Research (NWO)
} 
Consider two graphs $\Gamma$ and $\Gamma^{\prime}$ with adjacency matrices $A$ and $A^{\prime}$, respectively. The graphs are called cospectral if $A$ and $A^{\prime}$ have the same spectrum. If $A+y J$ and $A^{\prime}+y J$ (where $J$ is the all-one matrix) have the same spectrum for every $y \in \mathbb{R}$, then $\Gamma$ and $\Gamma^{\prime}$ are called $\mathbb{R}$-cospectral. Since $A$ and $A^{\prime}$ are symmetric, $\Gamma$ and $\Gamma^{\prime}$ are cospectral precisely when $A$ and $A^{\prime}$ are similar, that is, there exists an orthogonal matrix $Q$ such that $Q^{\top} A Q=A^{\prime}$. If $Q$ is a permutation matrix (i.e. $Q$ is regular of level 1 ) then $\Gamma$ and $\Gamma^{\prime}$ are isomorphic. If $\Gamma$ and $\Gamma^{\prime}$ are nonisomorphic, and there exist a regular orthogonal matrix $Q$ of level 2 such that $Q^{\top} A Q=A^{\prime}$, we call $\Gamma$ and $\Gamma^{\prime}$ semi-isomorphic. It easily follows that $\Gamma$ and $\Gamma^{\prime}$ are $\mathbb{R}$-cospectral if $Q$ is regular. (Indeed, $Q^{\top} \mathbf{1}=\mathbf{1}$ implies $\left.Q^{\top}(A+y J) Q=A^{\prime}+y J\right)$. In particular, semi-isomorphic graphs are $\mathbb{R}$-cospectral. By taking $y=-1$ we see that $\mathbb{R}$-cospectral graphs have cospectral complements. The following result, due to Johnson and Newman [3] (see also [1, 4]) states that the converse of some of these observations is also true.

Theorem 1. If $\Gamma$ and $\Gamma^{\prime}$ are graphs with adjacency matrices $A$ and $A^{\prime}$, respectively, then the following are equivalent.

$i$. The graphs $\Gamma$ and $\Gamma^{\prime}$ are cospectral, and so are their complements.

ii. The graphs $\Gamma$ and $\Gamma^{\prime}$ are $\mathbb{R}$-cospectral.

iii. There exists a regular orthogonal matrix $Q$, such that $Q^{\top} A Q=A^{\prime}$.

Any matrix of the form $x A+y J+z I$ with $x, y, z \in \mathbb{R}, x \neq 0$ is called a generalized adjacency matrix of $\Gamma$. Clearly $\Gamma$ and $\Gamma^{\prime}$ are $\mathbb{R}$-cospectral if and only if for every $x, y, z$ the corresponding generalized adjacency matrices have the same spectrum.

A graph $\Gamma$ is said to be determined by its spectrum if every graph cospectral with $\Gamma$ is isomorphic with $\Gamma$. A graph $\Gamma$ is determined by its generalized spectrum if every graph which is $\mathbb{R}$-cospectral with $\Gamma$ is isomorphic with $\Gamma$. It has been conjectured by the second author that almost every graph is determined by its spectrum. A weaker version states that almost every graph is determined by its generalized spectrum. Both conjectures are still open, but Wang and $\mathrm{Xu}$ [8] have a number of results supporting these conjectures. They prove that for almost no graph there exists a graph semi-isomorphic with it, and in addition they provide experimental evidence showing that a positive fraction of all pairs of nonisomorphic $\mathbb{R}$-cospectral graphs, are in fact semi-isomorphic. This makes it interesting to investigate semi-isomorphism.

In this paper we show how semi-isomorphic graphs can be made by a switching procedure, that generalizes the switching method due to Godsil and McKay [5] (see also [1, 6]), called GM-switching. We start with the classification of indecomposable regular orthogonal matrices of level 2, and then consider the generalized switching for the case that $Q$ has one nontrivial indecomposable block of order $4,6,7$ or 8 . In terms of the graph $\Gamma$ it means that $\Gamma$ must have a subgraph $\Delta$ of one of the mentioned orders that satisfies a number of properties. The four vertex case corresponds to GM-switching and the required properties are easily described; see Section 2. If $\Delta$ has six or seven vertices the required properties are worked out in detail. For eight vertices we restrict to the case $\Delta=\Gamma$.

As an application we determine all new switchings for graphs with eight vertices. We find 68 graphs for which GM-switching does not work, but the new switching does. It 
turns out that there exist only 22 pairs of $\mathbb{R}$-cospectral graphs on eight vertices which are not semi-isomorphic with each other or with another graph.

\section{Switching}

The following lemma describes the switching method of Godsil and McKay [5].

Lemma 2. Let $\Gamma$ be a graph and let $\left\{V_{1}, \ldots, V_{m}, W\right\}$ be a partition of the vertex set of $\Gamma$. Suppose that for every vertex $x \in W$ and every $i \in\{1, \ldots, m\}, x$ has either $0, \frac{1}{2}\left|V_{i}\right|$ or $\left|V_{i}\right|$ neighbors in $V_{i}$. Moreover, suppose that for every $i, j \in\{1, \ldots, m\}$ the number of neighbors in $V_{j}$ of a vertex $x \in V_{i}$ only depends on $i$ and $j$ (in other words, $\left\{V_{1}, \ldots, V_{m}\right\}$ is an equitable partition of $\Gamma \backslash W)$. Make a new graph $\Gamma^{\prime}$ as follows. For each $x \in W$ and $i \in\{1, \ldots, m\}$ such that $x$ has $\frac{1}{2}\left|V_{i}\right|$ neighbours in $V_{i}$ delete the corresponding $\frac{1}{2}\left|V_{i}\right|$ edges and join $x$ instead to the $\frac{1}{2}\left|V_{i}\right|$ other vertices in $V_{i}$. Then $\Gamma$ and $\Gamma^{\prime}$ are $\mathbb{R}$-cospectral.

Proof. Let $A$ and $A^{\prime}$ be the adjacency matrices of $\Gamma$ and $\Gamma^{\prime}$, respectively (the vertex ordering is assumed to be in accordance with the partition). Let $n$ be the number of vertices of $\Gamma$ and $\Gamma^{\prime}$. For $i=1, \ldots, m$ define the $\left|V_{i}\right| \times\left|V_{i}\right|$ matrix $R_{i}=\frac{2}{\left|V_{i}\right|} J-I$, and the $n \times n$ block diagonal matrix $Q=\operatorname{diag}\left(R_{1}, \ldots, R_{m}, I\right)$. Then $Q$ is orthogonal and regular, and it follows straightforwardly that $Q^{\top} A Q=A^{\prime}$, and more generally, that $Q^{\top}(A+y J) Q=A^{\prime}+y J$ for every $y \in \mathbb{R}$.

Note that the orthogonal matrix $Q$ used in the above proof is regular of level $\operatorname{lcm}\left(\left|V_{1}\right|, \ldots,\left|V_{m}\right|\right) / 2$. If $\left|V_{i}\right|=2$ for some $i \in\{1, \ldots, m\}$, then GM-switching just interchanges the two vertices of $V_{i}$, and therefore the two vertices may be considered part of $W$. Thus we can assume that $\left|V_{i}\right| \geqslant 4$. If $\left|V_{i}\right|=4$ for all $i \in\{1, \ldots, m\}$, then $Q$ has level 2, and the graphs $\Gamma$ and $\Gamma^{\prime}$ are semi-isomorphic, provided they are not isomorphic. The conditions for GM-switching are most easy to fulfill if $m=1$ and $\left|V_{1}\right|=4$. In this case the orthogonal matrix $Q$ is regular of level 2 and has just one nontrivial indecomposable block $R_{1}=\frac{1}{2} J-I$. For this switching to work, $V_{1}$ must induce a regular graph on four vertices, and each vertex outside $V_{1}$ should be adjacent to 0,2 , or 4 vertices of $V_{1}$. For example, the adjacency matrix $A$ given below satisfies these conditions, and $A^{\prime}$ is obtained by GM-switching: $A^{\prime}=Q^{\top} A Q$. Therefore the two graphs are $\mathbb{R}$-cospectral. The graphs are not isomorphic (because of different degree sequences), and therefore they are semi-isomorphic.

$$
A=\left[\begin{array}{llll|lll}
0 & 0 & 0 & 0 & 1 & 1 & 1 \\
0 & 0 & 0 & 0 & 1 & 0 & 0 \\
0 & 0 & 0 & 0 & 0 & 1 & 0 \\
0 & 0 & 0 & 0 & 0 & 0 & 1 \\
\hline 1 & 1 & 0 & 0 & 0 & 0 & 0 \\
1 & 0 & 1 & 0 & 0 & 0 & 0 \\
1 & 0 & 0 & 1 & 0 & 0 & 0
\end{array}\right], A^{\prime}=\left[\begin{array}{llll|lll}
0 & 0 & 0 & 0 & 0 & 0 & 0 \\
0 & 0 & 0 & 0 & 0 & 1 & 1 \\
0 & 0 & 0 & 0 & 1 & 0 & 1 \\
0 & 0 & 0 & 0 & 1 & 1 & 0 \\
\hline 0 & 0 & 1 & 1 & 0 & 0 & 0 \\
0 & 1 & 0 & 1 & 0 & 0 & 0 \\
0 & 1 & 1 & 0 & 0 & 0 & 0
\end{array}\right]
$$


This is the situation we will generalize. If $R$ is an indecomposable regular orthogonal $r \times r$ matrix of level 2 , and $\Gamma$ is a graph with $n \geqslant r$ vertices and adjacency matrix $A$. We define the $n \times n$ matrix

$$
Q=\left[\begin{array}{ll}
R & O \\
O & I
\end{array}\right]
$$

and investigate the required structure for $A$ needed to ensure that $A^{\prime}=Q^{\top} A Q$ is again the adjacency matrix of a graph. Note that it is sufficient to require that $A^{\prime}$ is a $(0,1)$ matrix, because $A^{\prime}$ is symmetric and trace $A^{\prime}=\operatorname{trace} A=0$.

\section{Regular orthogonal matrices of level 2}

Let $Q$ be a regular orthogonal matrix of level 2. Then after suitable reordering of rows and columns, $Q$ takes the block diagonal form $\operatorname{diag}\left(R_{1}, \ldots, R_{m}\right)$, or $\operatorname{diag}\left(R_{1}, \ldots, R_{m}, I\right)$, where $R_{i}$ is an indecomposable regular orthogonal matrix of level 2 for $i=1, \ldots, m$. It follows easily that if $R$ is an indecomposable regular orthogonal matrix of level 2 , then all entries of $2 R$ are equal to 0,1 or -1 , and each row and column of $R$ has exactly three 1 's and one -1 . Using these observations and the orthogonality of $R$, Wang and $\mathrm{Xu}[8,7]$ determined all indecomposable regular orthogonal matrices of level 2.

Theorem 3. Let $R$ be an indecomposable regular orthogonal matrix with level 2 and row sum 1 . Then after suitable reordering of rows and columns $R$ is one of the following:

$$
\begin{gathered}
\text { (i) } \frac{1}{2}\left[\begin{array}{rrrr}
-1 & 1 & 1 & 1 \\
1 & -1 & 1 & 1 \\
1 & 1 & -1 & 1 \\
1 & 1 & 1 & -1
\end{array}\right], \text { (ii) } \frac{1}{2}\left[\begin{array}{rrrrrr}
J & O & \cdots & \cdots & O & Y \\
Y & J & O & \cdots & \cdots & O \\
O & Y & J & O & \cdots & O \\
& \ddots & \ddots & \ddots & \ddots & \\
O & \cdots & O & Y & J & O \\
O & \cdots & \cdots & O & Y & J
\end{array}\right], \\
\text { (iii) } \frac{1}{2}\left[\begin{array}{rrrrrrr}
-1 & 1 & 1 & 0 & 1 & 0 & 0 \\
0 & -1 & 1 & 1 & 0 & 1 & 0 \\
0 & 0 & -1 & 1 & 1 & 0 & 1 \\
1 & 0 & 0 & -1 & 1 & 1 & 0 \\
0 & 1 & 0 & 0 & -1 & 1 & 1 \\
1 & 0 & 1 & 0 & 0 & -1 & 1 \\
1 & 1 & 0 & 1 & 0 & 0 & -1
\end{array}\right],(\text { iv }) \frac{1}{2}\left[\begin{array}{rrrrr}
-I & I & I & I \\
I & -Z & I & Z \\
I & Z & -Z & I \\
I & I & Z & -Z
\end{array}\right],
\end{gathered}
$$

where $I, J, O, Y=2 I-J$ and $Z=J-I$, are square matrices of order 2 .

We observed that $W=2 R$ is a matrix with entries 0,1 and -1 , satisfying $W W^{\top}=4 I$, and $W \mathbf{1}=W^{\top} \mathbf{1}=2 \cdot \mathbf{1}$. Such a matrix $W$ is known as a regular weighing matrix of weight 4 . Two weighing matrices are called equivalent if one can be obtained by the other 
by row and column permutations and/or multiplication of a number of rows and columns by -1 . The inequivalent weighing matrices of weight 4 have been classified in 1986 by Chan, Rodger and Seberry [2], and from their result the classification of the regular ones is straightforward. Therefore, Theorem 3 should be attributed to the authors of [2].

Case $(i i)$ of the above theorem gives an infinite family of matrices of even order starting with order 6 . So for the order 8 there exist two different indecomposable regular orthogonal matrices of level 2. If

$$
Q=\left[\begin{array}{ll}
R & O \\
O & I
\end{array}\right]
$$

and $R$ is as in case $(i)$, then the transformation $A^{\prime}=Q^{\top} A Q$ corresponds to GM-switching. In the next sections we will investigate the required structure for $A$ for the other three cases.

The product of two regular orthogonal matrices of level 2 is again a regular orthogonal matrix, but the level need not be 2 , but can also be 1 or 4 . Therefore we may not conclude that the relation: 'being isomorphic or semi-isomorphic' is an equivalence relation. In fact, this is false. This is illustrated by the following example.

Example 4. Consider the three nonisomorphic graphs $\Gamma, \Gamma_{1}, \Gamma_{2}$ with adjacency matrices

$$
A=\left[\begin{array}{llllllll}
0 & 0 & 0 & 0 & 1 & 1 & 0 & 0 \\
0 & 0 & 0 & 0 & 1 & 0 & 1 & 0 \\
0 & 0 & 0 & 0 & 0 & 1 & 1 & 1 \\
0 & 0 & 0 & 0 & 0 & 0 & 0 & 1 \\
1 & 1 & 0 & 0 & 0 & 0 & 0 & 1 \\
1 & 0 & 1 & 0 & 0 & 0 & 0 & 1 \\
0 & 1 & 1 & 0 & 0 & 0 & 0 & 1 \\
0 & 0 & 1 & 1 & 1 & 1 & 1 & 0
\end{array}\right], A_{1}=\left[\begin{array}{llll|llll}
0 & 0 & 0 & 0 & 0 & 0 & 1 & 1 \\
0 & 0 & 0 & 0 & 0 & 1 & 0 & 1 \\
0 & 0 & 0 & 0 & 1 & 0 & 0 & 0 \\
0 & 0 & 0 & 0 & 1 & 1 & 1 & 0 \\
\hline 0 & 0 & 1 & 1 & 0 & 0 & 0 & 1 \\
0 & 1 & 0 & 1 & 0 & 0 & 0 & 1 \\
1 & 0 & 0 & 1 & 0 & 0 & 0 & 1 \\
1 & 1 & 0 & 0 & 1 & 1 & 1 & 0
\end{array}\right], A_{2}=\left[\begin{array}{lll|llll|l}
0 & 0 & 0 & 1 & 0 & 0 & 1 & 0 \\
0 & 0 & 0 & 1 & 0 & 1 & 0 & 0 \\
0 & 0 & 0 & 1 & 1 & 0 & 0 & 1 \\
\hline 1 & 1 & 1 & 0 & 0 & 0 & 0 & 1 \\
0 & 0 & 1 & 0 & 0 & 0 & 0 & 1 \\
0 & 1 & 0 & 0 & 0 & 0 & 0 & 1 \\
1 & 0 & 0 & 0 & 0 & 0 & 0 & 1 \\
\hline 0 & 0 & 1 & 1 & 1 & 1 & 1 & 0
\end{array}\right] .
$$

The graphs $\Gamma_{1}$ and $\Gamma_{2}$ can both be obtained from $\Gamma$ by GM-switching. Therefore $\Gamma_{1}$ and $\Gamma_{2}$ are both semi-isomorphic with $\Gamma$. The regular orthogonal matrices that represent the switching are (with $R$ as in Case $(i)$ of Theorem 3 ):

$$
Q_{1}=Q_{1}^{\top}=\left[\begin{array}{cc}
R & O \\
O & I_{4}
\end{array}\right] \text {, and } Q_{2}=Q_{2}^{\top}=\left[\begin{array}{ccc}
I_{3} & O & \mathbf{0} \\
O & R & \mathbf{0} \\
\mathbf{0}^{\top} & \mathbf{0}^{\top} & 1
\end{array}\right] \text {. }
$$

Clearly, $Q=Q_{1} Q_{2}$ is orthogonal and regular and satisfies $Q^{\top} A_{1} Q=A_{2}$. But $Q$ has level 4. Moreover, it has been checked (by computer) that there exists no orthogonal regular $Q$ of level 2 for which $Q^{\top} A_{1} Q=A_{2}$. Therefore, $\Gamma_{1}$ and $\Gamma_{2}$ are not semi-isomorphic.

In some cases the product of two regular orthogonal matrices $Q_{1}$ and $Q_{2}$ of level 2 has level 2 again. This is obviously the case, if the rows of the nontrivial indecomposable blocks of $Q_{1}$ are all different from the rows of the nontrivial indecomposable blocks of $Q_{2}$. A nontrivial example is given by:

$$
Q_{1}=Q_{1}^{\top}=\left[\begin{array}{cc}
R_{2} & O \\
O & I_{2}
\end{array}\right], \text { and } Q_{2}=Q_{2}^{\top}=\left[\begin{array}{cc}
I & O \\
O & R_{1}
\end{array}\right]
$$


with $R_{1}$ as in Case $(i)$, and $R_{2}$ as in Case $(i)$ or $(i i)$ of Theorem 3. Then $Q_{1} Q_{2}$ has again level 2 and belongs to Case (ii) of Theorem 3. In case both $R_{1}$ and $R_{2}$ belong to Case $(i)$, then $Q_{1} Q_{2}$ correspond to a six vertex switching of Case $(i i)$. This shows that the six vertex switching, can sometimes be obtained by applying GM-switching twice.

\section{Six vertex switching}

Here we consider switching with a regular orthogonal matrix $Q$ of order $n$, having just one nontrivial indecomposable block of order 6 . Thus with a suitable ordering of rows and columns we have:

$$
Q=\left[\begin{array}{ll}
R & O \\
O & I
\end{array}\right], \text { where } R=\frac{1}{2}\left[\begin{array}{ccc}
J & O & Y \\
Y & J & O \\
O & Y & J
\end{array}\right] \text {, and } Y=\left[\begin{array}{rr}
1 & -1 \\
-1 & 1
\end{array}\right]
$$

Let $\Gamma$ be a graph with $n$ vertices and adjacency matrix

$$
A=\left[\begin{array}{cc}
B & V \\
V^{\top} & C
\end{array}\right]
$$

where $B$ is the adjacency matrix of a graph $\Delta$ of order 6 . For the six vertex switching with respect to $\Delta$ to work we need that the switched matrix

$$
A^{\prime}=Q^{\top} A Q=\left[\begin{array}{cc}
R^{\top} B R & R^{\top} V \\
V^{\top} R & C
\end{array}\right]
$$

is a $(0,1)$ matrix again. First we determine the possible columns of $V$. This means that we have to find the vectors $\mathbf{v} \in\{0,1\}^{6}$ for which $R^{\top} \mathbf{v}$ is again a $(0,1)$ vector.

Lemma 5. Let $\mathbf{v} \in\{0,1\}^{6}$. With $R$ as above, $R_{\mathbf{v}}^{\top} \in\{0,1\}^{6}$ if and only if the number of ones in each class of the partition is even, or the number of ones in each class of the partition is odd. In the first case $R^{\top} \mathbf{v}=\mathbf{v}$. In the second case, multiplication by $R^{\top}$ gives a permutation of the eight involved $(0,1)$ vectors represented by the following two cycles ([101010] and [010101] are fixed):

$$
\left([101001]^{\top},[100110]^{\top},[011010]^{\top}\right)\left([100101]^{\top},[010110]^{\top},[011001]^{\top}\right) \text {. }
$$

Proof. With $\mathbf{v}=\left[v_{1} \ldots v_{6}\right]^{\top}$ we have

$$
\mathbf{0}=2 R \mathbf{v}=\left[\begin{array}{c}
v_{1}+v_{2}+v_{3}-v_{4} \\
v_{1}+v_{2}-v_{3}+v_{4} \\
v_{3}+v_{4}+v_{5}-v_{6} \\
v_{3}+v_{4}-v_{5}+v_{6} \\
v_{1}-v_{2}+v_{5}+v_{6} \\
-v_{1}+v_{2}+v_{5}+v_{6}
\end{array}\right]=\left[\begin{array}{c}
v_{1,2}+v_{3,4} \\
v_{1,2}+v_{3,4} \\
v_{3,4}+v_{5,6} \\
v_{3,4}+v_{5,6} \\
v_{1,2}+v_{5,6} \\
v_{1,2}+v_{5,6}
\end{array}\right] \quad(\bmod 2)
$$


where $v_{i, i+1}=v_{i}+v_{i+1}$ for $i=1,3,5$. It follows that $R^{\top} \mathbf{v}$ is a $(0,1)$ vector if and only if $v_{1,2}=v_{3,4}=v_{5,6}(\bmod 2)$. The second part of the lemma follows by straightforward verification.

Next we determine the set $\mathcal{B}$ of adjacency matrices $B$ of order 6 , that have the property that $B^{\prime}=R^{\top} B R$ is a $(0,1)$ matrix again. To do so, the following observations are useful. The matrix $R$ is invariant under certain reorderings of rows and columns, more precisely: $R=P^{\top} R P$, when $P$ is any permutation matrix generated by

$$
P_{1}=\left[\begin{array}{ccc}
O & I & O \\
O & O & I \\
I & O & O
\end{array}\right] \text { and } P_{2}=\left[\begin{array}{ccc}
Z & O & O \\
O & Z & O \\
O & O & Z
\end{array}\right], \text { where } Z=\left[\begin{array}{cc}
0 & 1 \\
1 & 0
\end{array}\right] .
$$

Clearly, $B^{\prime}=R^{\top} B R$ implies $P^{\top} B^{\prime} P=R^{\top}\left(P^{\top} B P\right) R$, so $\mathcal{B}$ is invariant under the mentioned permutations and $\left(P^{\top} B P\right)^{\prime}=P^{\top} B^{\prime} P$. Moreover, $B^{\prime}=R^{\top} B R$ implies $J-B^{\prime}-I=R^{\top}(J-$ $B-I) R$, so $\mathcal{B}$ is also invariant under taking complements and $(J-B-I)^{\prime}=J-B^{\prime}-I$. But there is more. The permutation matrix $P_{2}$ commutes with $R$, and therefore $P_{2}+B^{\prime}=$ $R^{\top}\left(P_{2}+B\right) R$, so if $B \in \mathcal{B}$, and the three diagonal blocks of $B$ are $O$, then $B+P_{2} \in \mathcal{B}$ and $\left(P_{2}+B\right)^{\prime}=P_{2}+B^{\prime}$.

Lemma 6. Let $B$ be an adjacency matrix of order six. With $R$ as above, the matrix $B^{\prime}=R^{\top} B R$ is again an adjacency matrix if and only if $B$ can be obtained from one of the following $B_{0} \ldots B_{7}$ by the above mentioned operations.

$$
\begin{gathered}
B_{0}=O, B_{1}=\left[\begin{array}{ccc}
O & J & O \\
J & O & O \\
O & O & O
\end{array}\right], B_{2}=\left[\begin{array}{ccc}
O & I & I \\
I & O & I \\
I & I & O
\end{array}\right], B_{3}=\left[\begin{array}{ccc}
O & I & I \\
I & O & Z \\
I & Z & O
\end{array}\right], \\
B_{4}=\left[\begin{array}{ccc}
O & N & N^{\top} \\
N^{\top} & O & N \\
N & N^{\top} & O
\end{array}\right], B_{5}=\left[\begin{array}{ccc}
O & M & N^{\top} \\
M^{\top} & O & N \\
N & N^{\top} & O
\end{array}\right], B_{6}=\left[\begin{array}{ccc}
O & O & I \\
O & O & M \\
I & M^{\top} & O
\end{array}\right], B_{7}=\left[\begin{array}{ccc}
O & O & Z \\
O & O & N \\
Z & N^{\top} & O
\end{array}\right],
\end{gathered}
$$

where $N=\left[\begin{array}{ll}0 & 0 \\ 1 & 1\end{array}\right], M=J-N$ and $Z=J-I$. The switched matrices $B_{i}^{\prime}=R^{\top} B_{i} R$ are:

$$
\begin{gathered}
B_{0}^{\prime}=O, B_{1}^{\prime}=B_{1}, B_{2}^{\prime}=B_{2}, B_{3}^{\prime}=\left[\begin{array}{ccc}
O & I & Z \\
I & O & I \\
Z & I & O
\end{array}\right], \\
B_{4}^{\prime}=\left[\begin{array}{ccc}
O & N^{\top} & N \\
N & O & N^{\top} \\
N^{\top} & N & O
\end{array}\right], B_{5}^{\prime}=\left[\begin{array}{ccc}
O & N^{\top} & N \\
N & O & M^{\top} \\
N^{\top} & M & O
\end{array}\right], B_{6}^{\prime}=\left[\begin{array}{ccc}
O & O & M \\
O & O & I \\
M^{\top} & I & O
\end{array}\right], B_{7}^{\prime}=\left[\begin{array}{ccc}
O & O & N \\
O & O & Z \\
N^{\top} & Z & O
\end{array}\right] .
\end{gathered}
$$


Proof. With the vertex ordering used for $R$, we write

$$
B=\left[\begin{array}{ccc}
B_{1,1} & B_{1,2} & B_{1,3} \\
B_{2,1} & B_{2,2} & B_{2,3} \\
B_{3,1} & B_{3,2} & B_{3,3}
\end{array}\right] \text {, and } B^{\prime}=R^{\top} B R=\left[\begin{array}{ccc}
B_{1,1}^{\prime} & B_{1,2}^{\prime} & B_{1,3}^{\prime} \\
B_{2,1}^{\prime} & B_{2,2}^{\prime} & B_{2,3}^{\prime} \\
B_{3,1}^{\prime} & B_{3,2}^{\prime} & B_{3,3}^{\prime}
\end{array}\right]
$$

This leads to

$$
4 B_{i, i}^{\prime}=J B_{i, i} J+J B_{i, i+1} Y+Y B_{i+1, i} J+Y B_{i+1, i+1} Y,
$$

for $i=1,2,3$ (addition $\bmod 3)$, where $B_{i, j}=B_{j, i}^{\top}$. Without loss of generality we take $B_{1,1}=O$. Taking traces in Equation 1 yields trace $\left(Y B_{2,2} Y\right)=0$, and therefore $B_{2,2}=O$. Thus $B_{i, i}=O$ for $i=1,2,3$. Equation 1 becomes $4 B_{i, i}^{\prime}=J B_{i, i+1} Y+\left(J B_{i, i+1} Y\right)^{\top}$. For every $2 \times 2$ matrix $X, J X Y=\alpha(M-N)$ for some scalar $\alpha$. Since $B_{i, i}^{\prime}$ has no negative entries it follows that $\alpha=0$ when $X=B_{i, i+1}$. Therefore $J B_{i, i+1} Y=O$, which reflects that $B_{i, i+1}$ has constant column sums for $i=1,2,3$. Equivalently, $B_{i, i+2}=B_{i+2, i}^{\top}=B_{i+2, i+3}^{\top}$ has constant row sums for $i=1,2,3$. Now it is straightforward to find all admissible matrices $B$ and the corresponding $B^{\prime}$.

For example the following matrix $A$ has the desired form (indeed, $B=B_{4}+P_{2}$ and $V$ has columns $[001100]^{\top}$ and $\left.[101001]^{\top}\right)$. With the above Lemmas we conclude that the switched matrix $A^{\prime}$ is cospectral with $A$.

$$
A=\left[\begin{array}{llllll|ll}
0 & 1 & 0 & 0 & 0 & 1 & 0 & 1 \\
1 & 0 & 1 & 1 & 0 & 1 & 0 & 0 \\
0 & 1 & 0 & 1 & 0 & 0 & 1 & 1 \\
0 & 1 & 1 & 0 & 1 & 1 & 1 & 0 \\
0 & 0 & 0 & 1 & 0 & 1 & 0 & 0 \\
1 & 1 & 0 & 1 & 1 & 0 & 0 & 1 \\
\hline 0 & 0 & 1 & 1 & 0 & 0 & 0 & 0 \\
1 & 0 & 1 & 0 & 0 & 1 & 0 & 0
\end{array}\right], A^{\prime}=\left[\begin{array}{llllll|ll}
0 & 1 & 0 & 1 & 0 & 0 & 0 & 1 \\
1 & 0 & 0 & 1 & 1 & 1 & 0 & 0 \\
0 & 0 & 0 & 1 & 0 & 1 & 1 & 0 \\
1 & 1 & 1 & 0 & 0 & 1 & 1 & 1 \\
0 & 1 & 0 & 0 & 0 & 1 & 0 & 1 \\
0 & 1 & 1 & 1 & 1 & 0 & 0 & 0 \\
\hline 0 & 0 & 1 & 1 & 0 & 0 & 0 & 0 \\
1 & 0 & 0 & 1 & 1 & 0 & 0 & 0
\end{array}\right] .
$$

The two graphs are not isomorphic, because the degree sequences differ, but they are semi-isomorphic. In addition, it has been verified by computer that the graphs are not related by GM-switching.

Out of the eight adjacency matrices presented in Lemma 6 , the graphs with matrices $B_{4}$ and $B_{5}$ are isomorphic, and the same is true for $B_{6}$ and $B_{7}$. In addition, the complement of $B_{4}$ (and $B_{5}$ ) is isomorphic with $B_{4}+P_{2}$, and the complement of $B_{2}$ is isomorphic with $B_{3}+P_{2}$. Therefore, the total number of nonisomorphic graphs $\Delta$ for which the six vertex switching works is 18 . The total number of matrices $B$ for which $R^{\top} B R$ is a $(0,1)$ matrix equals 96 .

We note that in Lemma 6 in all cases the graph $\Delta^{\prime}$ with matrix $B^{\prime}$ is isomorphic to $\Delta$ with matrix $B$. This implies that with a suitable reordering of the rows and columns of $R$ we can establish that $B^{\prime}=B$. However, this would require a reordering of the entries of the vectors in Lemma 5 depending on the choice of $B$. So it would not have made the presentation easier. Besides that, the phenomenon is not general, as we shall see in the next section. 


\section{$5 \quad$ Seven vertex switching}

Here we consider switching with a regular orthogonal matrix $Q$ of order $n$, having just one nontrivial indecomposable block $R$ of order 7 . Theorem 3 gives

$$
R=\frac{1}{2}\left[\begin{array}{rrrrrrr}
-1 & 1 & 1 & 0 & 1 & 0 & 0 \\
0 & -1 & 1 & 1 & 0 & 1 & 0 \\
0 & 0 & -1 & 1 & 1 & 0 & 1 \\
1 & 0 & 0 & -1 & 1 & 1 & 0 \\
0 & 1 & 0 & 0 & -1 & 1 & 1 \\
1 & 0 & 1 & 0 & 0 & -1 & 1 \\
1 & 1 & 0 & 1 & 0 & 0 & -1
\end{array}\right]
$$

Let $\Gamma$ be a graph with $n$ vertices and adjacency matrix

$$
A=\left[\begin{array}{cc}
B & V \\
V^{\top} & C
\end{array}\right]
$$

where now $B$ is the adjacency matrix of a graph $\Delta$ with seven vertices. For the seven vertex switching with respect to $\Delta$ to work we need that the switched matrix

$$
A^{\prime}=Q^{\top} A Q=\left[\begin{array}{cc}
R^{\top} B R & R^{\top} V \\
V^{\top} R & C
\end{array}\right]
$$

is a $(0,1)$ matrix again. Note that the matrix $R$ is invariant under a cyclic shift, that is, $P_{1} R P_{1}^{\top}=R$ for the cyclic permutation matrix $P_{1}=\operatorname{cycle}(0,1,0,0,0,0,0)$. Moreover, also the following permutation leaves $R$ invariant:

$$
P_{2}=\left[\begin{array}{lllllll}
1 & 0 & 0 & 0 & 0 & 0 & 0 \\
0 & 0 & 1 & 0 & 0 & 0 & 0 \\
0 & 0 & 0 & 0 & 1 & 0 & 0 \\
0 & 0 & 0 & 0 & 0 & 0 & 1 \\
0 & 1 & 0 & 0 & 0 & 0 & 0 \\
0 & 0 & 0 & 1 & 0 & 0 & 0 \\
0 & 0 & 0 & 0 & 0 & 1 & 0
\end{array}\right]
$$

Thus the permutation group $G$ generated by $P_{1}$ and $P_{2}$ is an automorphism group of $R$.

Remark. The group $G$ is known as the Frobenius group $F_{7,3}$, which can be described as the additive group of the field $\mathbb{F}_{7}$ extended with the multiplications by a nonzero square. It is the automorphism group of $R$, but also an automorphism group of the Fano plane. Indeed, $2 R+I$, and also $J-2 R-2 I$ are incidence matrices of the Fano plane.

First we determine the possible columns of $V$. This means that we have to find the vectors $\mathbf{v} \in\{0,1\}^{7}$ for which $R^{\top} \mathbf{v}$ is again a $(0,1)$ vector.

Lemma 7. Let $\mathbf{v} \in\{0,1\}^{7}$. With $R$ and $P_{1}$ as above, $R^{\top} \mathbf{v} \in\{0,1\}^{7}$ if and only if the vector $\mathbf{v}$ or the complement $\mathbf{1}-\mathbf{v}$ is equal to $\mathbf{0}$, or $P_{1}^{i}[1101000]^{\top}$ for some $i \in\{0, \ldots, 6\}$. If $\mathbf{v}=P_{1}^{i}[1101000]^{\top}$, or $P_{1}^{i}[0010111]^{\top}$, then $R^{\top} \mathbf{v}=P_{1}^{i}[0010110]^{\top}$, or $P_{1}^{i}[1101001]^{\top}$, respectively. 
Proof. This follows by straightforward verification. Using the above mentioned automorphisms of $R$, and the fact that $R^{\top}(\mathbf{1}-\mathbf{v})=\mathbf{1}-R^{\top} \mathbf{v}$, there are just a few cases to be checked.

Next we determine the set $\mathcal{B}$ of adjacency matrices $B$ of order 7 , that have the property that $B^{\prime}=R^{\top} B R$ is a $(0,1)$ matrix again. In the determination and description of $\mathcal{B}$ we use that $\mathcal{B}$ is invariant under the action of $G$, and under complementation. More precisely, if $B \in \mathcal{B}$, then so is $J-B-I$, and $P^{\top} B P$ for $P \in G$. Moreover, $(J-B-I)^{\prime}=$ $R^{\top}(J-B-I) R=J-B^{\prime}-I$ and $\left(P^{\top} B P\right)^{\prime}=R^{\top} P^{\top} B P R=P^{\top} B^{\prime} P$.

Lemma 8. Let $B$ be an adjacency matrix of order seven. With $R, P_{1}$ and $P_{2}$ as above, the matrix $B^{\prime}=R^{\top} B R$ is again an adjacency matrix if and only if $B$ can be obtained from one of the following $B_{0} \ldots B_{11}$ by complementation and/or a permutation of rows and columns generated by $P_{1}$ and $P_{2}$.

$$
\begin{aligned}
& B_{0}=O, B_{1}=\left[\begin{array}{lllllll}
0 & 0 & 0 & 1 & 1 & 0 & 0 \\
0 & 0 & 0 & 0 & 1 & 1 & 0 \\
0 & 0 & 0 & 0 & 0 & 1 & 1 \\
1 & 0 & 0 & 0 & 0 & 0 & 1 \\
1 & 1 & 0 & 0 & 0 & 0 & 0 \\
0 & 1 & 1 & 0 & 0 & 0 & 0 \\
0 & 0 & 1 & 1 & 0 & 0 & 0
\end{array}\right], B_{2}=\left[\begin{array}{lllllll}
0 & 0 & 0 & 0 & 0 & 0 & 1 \\
0 & 0 & 0 & 0 & 0 & 1 & 1 \\
0 & 0 & 0 & 1 & 0 & 1 & 1 \\
0 & 0 & 1 & 0 & 1 & 0 & 1 \\
0 & 0 & 0 & 1 & 0 & 0 & 0 \\
0 & 1 & 1 & 0 & 0 & 0 & 0 \\
1 & 1 & 1 & 1 & 0 & 0 & 0
\end{array}\right], \\
& B_{3}=\left[\begin{array}{lllllll}
0 & 0 & 0 & 0 & 0 & 1 & 1 \\
0 & 0 & 0 & 0 & 0 & 0 & 1 \\
0 & 0 & 0 & 1 & 0 & 1 & 1 \\
0 & 0 & 1 & 0 & 1 & 0 & 1 \\
0 & 0 & 0 & 1 & 0 & 1 & 0 \\
1 & 0 & 1 & 0 & 1 & 0 & 1 \\
1 & 1 & 1 & 1 & 0 & 1 & 0
\end{array}\right], B_{4}=\left[\begin{array}{lllllll}
0 & 0 & 0 & 0 & 0 & 0 & 1 \\
0 & 0 & 0 & 1 & 1 & 0 & 1 \\
0 & 0 & 0 & 0 & 0 & 0 & 0 \\
0 & 1 & 0 & 0 & 0 & 1 & 0 \\
0 & 1 & 0 & 0 & 0 & 1 & 1 \\
0 & 0 & 0 & 1 & 1 & 0 & 0 \\
1 & 1 & 0 & 0 & 1 & 0 & 0
\end{array}\right], B_{5}=\left[\begin{array}{lllllll}
0 & 0 & 0 & 0 & 1 & 1 & 0 \\
0 & 0 & 1 & 1 & 0 & 0 & 1 \\
0 & 1 & 0 & 1 & 0 & 0 & 1 \\
0 & 1 & 1 & 0 & 0 & 0 & 1 \\
1 & 0 & 0 & 0 & 0 & 1 & 0 \\
1 & 0 & 0 & 0 & 1 & 0 & 0 \\
0 & 1 & 1 & 1 & 0 & 0 & 0
\end{array}\right], \\
& B_{6}=\left[\begin{array}{lllllll}
0 & 0 & 0 & 1 & 0 & 0 & 1 \\
0 & 0 & 0 & 0 & 1 & 0 & 1 \\
0 & 0 & 0 & 0 & 0 & 0 & 0 \\
1 & 0 & 0 & 0 & 1 & 1 & 1 \\
0 & 1 & 0 & 1 & 0 & 1 & 1 \\
0 & 0 & 0 & 1 & 1 & 0 & 0 \\
1 & 1 & 0 & 1 & 1 & 0 & 0
\end{array}\right], B_{7}=\left[\begin{array}{lllllll}
0 & 0 & 0 & 0 & 0 & 0 & 0 \\
0 & 0 & 0 & 0 & 0 & 1 & 1 \\
0 & 0 & 0 & 1 & 0 & 1 & 0 \\
0 & 0 & 1 & 0 & 1 & 0 & 0 \\
0 & 0 & 0 & 1 & 0 & 0 & 1 \\
0 & 1 & 1 & 0 & 0 & 0 & 0 \\
0 & 1 & 0 & 0 & 1 & 0 & 0
\end{array}\right], B_{8}=\left[\begin{array}{lllllll}
0 & 0 & 0 & 0 & 0 & 0 & 1 \\
0 & 0 & 0 & 0 & 1 & 0 & 0 \\
0 & 0 & 0 & 1 & 1 & 0 & 1 \\
0 & 0 & 1 & 0 & 0 & 1 & 0 \\
0 & 1 & 1 & 0 & 0 & 0 & 0 \\
0 & 0 & 0 & 1 & 0 & 0 & 0 \\
1 & 0 & 1 & 0 & 0 & 0 & 0
\end{array}\right], \\
& B_{9}=\left[\begin{array}{lllllll}
0 & 0 & 0 & 0 & 0 & 0 & 1 \\
0 & 0 & 0 & 1 & 1 & 1 & 1 \\
0 & 0 & 0 & 0 & 0 & 1 & 0 \\
0 & 1 & 0 & 0 & 0 & 0 & 0 \\
0 & 1 & 0 & 0 & 0 & 1 & 1 \\
0 & 1 & 1 & 0 & 1 & 0 & 1 \\
1 & 1 & 0 & 0 & 1 & 1 & 0
\end{array}\right], B_{10}=\left[\begin{array}{lllllll}
0 & 0 & 0 & 0 & 0 & 1 & 0 \\
0 & 0 & 0 & 1 & 0 & 0 & 1 \\
0 & 0 & 0 & 1 & 1 & 1 & 1 \\
0 & 1 & 1 & 0 & 0 & 1 & 0 \\
0 & 0 & 1 & 0 & 0 & 0 & 1 \\
1 & 0 & 1 & 1 & 0 & 0 & 0 \\
0 & 1 & 1 & 0 & 1 & 0 & 0
\end{array}\right], B_{11}=\left[\begin{array}{lllllll}
0 & 0 & 0 & 1 & 0 & 0 & 1 \\
0 & 0 & 0 & 1 & 1 & 1 & 0 \\
0 & 0 & 0 & 0 & 1 & 1 & 1 \\
1 & 1 & 0 & 0 & 0 & 1 & 0 \\
0 & 1 & 1 & 0 & 0 & 0 & 0 \\
0 & 1 & 1 & 1 & 0 & 0 & 1 \\
1 & 0 & 1 & 0 & 0 & 1 & 0
\end{array}\right] .
\end{aligned}
$$

The switched matrices $B_{i}^{\prime}=R^{\top} B_{i} R$ satisfy $B_{0}^{\prime}=B_{0}, B_{1}^{\prime}=B_{1}, B_{i}^{\prime}=Z_{7} B_{i} Z_{7}$ for $i=$ $2, \ldots, 5, B_{6}^{\prime}=Z_{7} B_{9} Z_{7}, B_{9}^{\prime}=Z_{7} B_{6} Z_{7}, B_{10}^{\prime}=Z_{7} B_{2} Z_{7}$, and

$$
B_{7}^{\prime}=\left[\begin{array}{lllllll}
0 & 0 & 0 & 1 & 0 & 1 & 1 \\
0 & 0 & 0 & 0 & 0 & 1 & 0 \\
0 & 0 & 0 & 1 & 0 & 0 & 0 \\
1 & 0 & 1 & 0 & 0 & 0 & 0 \\
0 & 0 & 0 & 0 & 0 & 0 & 1 \\
1 & 1 & 0 & 0 & 0 & 0 & 0 \\
1 & 0 & 0 & 0 & 1 & 0 & 0
\end{array}\right], B_{8}^{\prime}=\left[\begin{array}{lllllll}
0 & 0 & 0 & 0 & 1 & 0 & 1 \\
0 & 0 & 0 & 1 & 1 & 0 & 0 \\
0 & 0 & 0 & 0 & 0 & 0 & 0 \\
0 & 1 & 0 & 0 & 0 & 1 & 0 \\
1 & 1 & 0 & 0 & 0 & 0 & 0 \\
0 & 0 & 0 & 1 & 0 & 0 & 1 \\
1 & 0 & 0 & 0 & 0 & 1 & 0
\end{array}\right], B_{11}^{\prime}=\left[\begin{array}{lllllll}
0 & 0 & 1 & 1 & 1 & 0 & 1 \\
0 & 0 & 0 & 1 & 1 & 0 & 0 \\
1 & 0 & 0 & 0 & 1 & 1 & 0 \\
1 & 1 & 0 & 0 & 0 & 0 & 1 \\
1 & 1 & 1 & 0 & 0 & 0 & 0 \\
0 & 0 & 1 & 0 & 0 & 0 & 1 \\
1 & 0 & 0 & 1 & 0 & 1 & 0
\end{array}\right],
$$

where $Z_{7}$ is the reverse identity matrix of order 7 , that is, $\left(Z_{7}\right)_{i, j}=1$ if $i+j=7$, and 0 otherwise. 
Again the proof goes by straightforward verification. Observe that $B_{0}$ to $B_{11}$ are all nonisomorphic, and together with the complements this gives 24 nonisomorphic graphs for which the seven vertex switching works. Out of these graphs $B_{0}$ and its complement are the only ones invariant under the group $G$. Of the remaining cases $B_{1}$ and its complement are invariant under the cyclic permutation $P_{1}$, and $B_{5}, \ldots, B_{9}$ and their complements are invariant under $P_{2}$. So in total there are 288 adjacency matrices $B$ of order 7 for which $B^{\prime}=R^{\top} B R$ is again an adjacency matrix. For the six vertex switching we observed that $B_{i}^{\prime}$ is isomorphic with $B_{i}$ in all cases. This is not true anymore for the seven vertex switching. Indeed, $B_{i}^{\prime}$ is nonisomorphic (and hence semi-isomorphic) to $B_{i}$ for $i=6, \ldots, 10$. It is not difficult to see that these semi-isomorphic pairs can also be made by GM-switching with respect to four vertices. However, the following example on eight vertices gives semiisomorphic graphs that can be made by the seven vertex switching described above, but not by GM-switching.

$$
A=\left[\begin{array}{lllllll|l}
0 & 0 & 0 & 0 & 0 & 1 & 1 & 1 \\
0 & 0 & 0 & 0 & 0 & 0 & 1 & 1 \\
0 & 0 & 0 & 1 & 0 & 1 & 1 & 0 \\
0 & 0 & 1 & 0 & 1 & 0 & 1 & 1 \\
0 & 0 & 0 & 1 & 0 & 1 & 0 & 0 \\
1 & 0 & 1 & 0 & 1 & 0 & 1 & 0 \\
1 & 1 & 1 & 1 & 0 & 1 & 0 & 0 \\
\hline 1 & 1 & 0 & 1 & 0 & 0 & 0 & 0
\end{array}\right], A^{\prime}=\left[\begin{array}{lllllll|l}
0 & 1 & 0 & 1 & 1 & 1 & 1 & 0 \\
1 & 0 & 1 & 0 & 1 & 0 & 1 & 0 \\
0 & 1 & 0 & 1 & 0 & 0 & 0 & 1 \\
1 & 0 & 1 & 0 & 1 & 0 & 0 & 0 \\
1 & 1 & 0 & 1 & 0 & 0 & 0 & 1 \\
1 & 0 & 0 & 0 & 0 & 0 & 0 & 1 \\
1 & 1 & 0 & 0 & 0 & 0 & 0 & 0 \\
\hline 0 & 0 & 1 & 0 & 1 & 1 & 0 & 0
\end{array}\right]
$$

\section{Eight vertex switching}

In this section we consider the case that $Q$ has one nontrivial indecomposable block $R$ of order 8 . Theorem 3 gives two nonequivalent possibilities for $R$, being:

$$
R_{1}=\frac{1}{2}\left[\begin{array}{cccc}
J & O & O & Y \\
Y & J & O & O \\
O & Y & J & O \\
O & O & Y & J
\end{array}\right] \text {, and } R_{2}=\frac{1}{2}\left[\begin{array}{rrrr}
-I & I & I & I \\
I & -Z & I & Z \\
I & Z & -Z & I \\
I & I & Z & -Z
\end{array}\right]
$$

with $I, J, O, Y=2 I-J$, and $Z=J-I$ of order 2. We had hoped to find a general description of matrices $B$ for which $R B R$ is a $(0,1)$ matrix again, when $R$ has the form of Case (ii) in Theorem 3, but failed. Already for the above matrix $R_{1}$ of order 8 , we found 3584 such matrices, and we were not able to discover a general structure. Also for $R_{2}$ we found a large number (1504) of such matrices $B$, so we decided not to give a complete description of the switching conditions as we did in the previous sections for six and seven vertex switching. However, in the next section we will investigate semi-isomorphism for graphs on eight vertices. Therefore we also have to consider eight vertex switching with no additional vertices, that is, $Q=R$. In this case we only have to consider adjacency matrices $B$ for which $B^{\prime}=R^{\top} B R$ is nonisomorphic with $B$. With the help of a computer we found the following: 


\begin{tabular}{|c|c|c|c|c|c|c|c|}
\hline 00110110 & 00111011 & 00011010 & 00101001 & 00111010 & 00101011 & 00110110 & 00111011 \\
\hline 00111010 & 00111000 & 00101010 & 00101010 & 00001010 & 00101000 & 00111010 & 00111000 \\
\hline 11000011 & 11000001 & 01001100 & 11001100 & 10001111 & 11001110 & 11001111 & 11001101 \\
\hline 11000000 & 11000010 & 10001100 & 00001100 & 10001100 & 00001110 & 11001100 & 11001110 \\
\hline 01000000 & 11000000 & 11110000 & 11110000 & 11110000 & 11110000 & 01110000 & 11110000 \\
\hline 10000000 & 00000000 & 00110000 & 00110000 & 00110000 & 00110000 & 10110000 & 00110000 \\
\hline 11100000 & 10010000 & 11000000 & 01000000 & 11100000 & 10110000 & 11100000 & 10010000 \\
\hline 00100000 & 10100000 & 00000000 & 10000000 & 00100000 & 10000000 & 00100000 & 10100000 \\
\hline 00110100 & 00111100 & 00111011 & 00101111 & 00110111 & 00111111 & 00110011 & 00010011 \\
\hline 00111000 & 00110000 & 00001011 & 00100011 & 00111011 & 00110011 & 00000011 & 00100011 \\
\hline 11001110 & 11001001 & 10001110 & 11001010 & 11001110 & 11001001 & 10000110 & 01001010 \\
\hline 11000010 & 11001010 & 10000010 & 00001010 & 11000010 & 11001010 & 10001010 & 10001010 \\
\hline 01100000 & 10110000 & 11100000 & 10110000 & 01100000 & 10110000 & 00010000 & 00110000 \\
\hline 10100000 & 10000000 & 00100000 & 10000000 & 10100000 & 10000000 & 00100000 & 00000000 \\
\hline 00110000 & 00010000 & 11110000 & 11110000 & 11110000 & 11010000 & 11110000 & 11110000 \\
\hline 00000000 & 00100000 & 11000000 & 11000000 & 11000000 & 11100000 & 11000000 & 11000000 \\
\hline 00111111 & 00011111 & 00011010 & 00101001 & & & & \\
\hline 00001111 & 00101111 & 00101010 & 00101010 & & & & \\
\hline 10000110 & 01001010 & 01001111 & 11001111 & & & & \\
\hline 10001010 & 10001010 & 10001111 & 00001111 & & & & \\
\hline 11010000 & 11110000 & 11110000 & 11110000 & & & & \\
\hline 11100000 & 11000000 & 00110000 & 00110000 & & & & \\
\hline 11110000 & 11110000 & 11110000 & 01110000 & & & & \\
\hline 11000000 & 11000000 & 00110000 & 10110000 & & & & \\
\hline
\end{tabular}

Table 1: Nonisomorphic pairs $B_{i}, B_{i}^{\prime}=R_{1}^{\top} B_{i} R_{1}(i=1, \ldots, 10)$ mentioned in Lemma 9.

Lemma 9. There exist exactly 20 nonisomorphic graphs $\Gamma_{1}, \ldots, \Gamma_{20}$, which have an adjacency matrix $B_{i}$ for which $B_{i}^{\prime}=R_{1}^{\top} B_{i} R_{1}$ is the adjacency matrix of a graph nonisomorphic with $\Gamma_{i}$ for $i=1, \ldots, 20$. The matrices $B_{1}, \ldots, B_{10}$ of $\Gamma_{1} \ldots \Gamma_{10}$ are displayed in Table 1 , and $\Gamma_{11}, \ldots, \Gamma_{20}$ are the complements of $\Gamma_{1}, \ldots, \Gamma_{10}$.

There exist exactly 36 nonisomorphic graphs $\Gamma_{21}, \ldots, \Gamma_{56}$, which have an adjacency matrix $B_{i}$ for which $B_{i}^{\prime}=R_{2}^{\top} B_{i} R_{2}$ is the adjacency matrix of a graph nonisomorphic with $\Gamma_{i}$ for $i=21, \ldots, 56$. The matrices $B_{21}, \ldots, B_{38}$ of $\Gamma_{21} \ldots \Gamma_{38}$ are displayed in Table 2 , and $\Gamma_{39}, \ldots, \Gamma_{56}$ are the complements of $\Gamma_{21}, \ldots, \Gamma_{36}$.

\section{Semi-isomorphic graphs with eight vertices}

With the results of the previous sections, we were able to generated by computer all graphs on eight vertices for which the six vertex switching (with two extra vertices), the seven vertex switching (with one extra vertex), or one of the eight vertex switching (with no extra vertex) applies and gives a nonisomorphic (and therefore semi-isomorphic) mate. In total we found 427 nonisomorphic graphs; 227 by six vertex switching, 144 by seven vertex switching, and 56 (see Lemma 9) by eight vertex switching.

For $n \leqslant 11$, Table 1 of [6] gives exact numbers of nonisomorphic graphs on $n$ vertices 


\begin{tabular}{|c|c|c|c|c|c|c|c|}
\hline 00001010 & 00111100 & 01100011 & 00111000 & 01001100 & 00011100 & 01110101 & 00001000 \\
\hline 00100110 & 00010000 & 10100110 & 00010000 & 10101010 & 00000000 & 10001100 & 00101100 \\
\hline 01011010 & 10011001 & 11011010 & 10011101 & 01001001 & 00010011 & 10010011 & 01011101 \\
\hline 00101100 & 11100000 & 00101100 & 11100000 & 00001010 & 10101010 & 10100110 & 00100011 \\
\hline 10110000 & 10100010 & 00110000 & 10100110 & 11110000 & 10010001 & 01000000 & 11100100 \\
\hline 01010000 & 10000001 & 01010000 & 00101011 & 10000000 & 10000010 & 11010000 & 01101010 \\
\hline 11100000 & 00001000 & 11100000 & 00001100 & 01010000 & 00110100 & 00110000 & 00010100 \\
\hline 00000000 & 00100100 & 10000000 & 00100100 & 00100000 & 00101000 & 10100000 & 00110000 \\
\hline 00011111 & 00101000 & 00111001 & 00101001 & 01011111 & 00101000 & 00001010 & 00111101 \\
\hline 00000100 & 00111010 & 00010010 & 00110010 & 10010010 & 00110010 & 00101110 & 00010000 \\
\hline 00010010 & 11011001 & 10010010 & 11011000 & 00010010 & 11011001 & 01010010 & 10011001 \\
\hline 10101100 & 01100001 & 11101100 & 01101001 & 11101100 & 01101001 & 00101100 & 11100001 \\
\hline 10010110 & 11100010 & 10010110 & 10110110 & 10010110 & 10110111 & 11010110 & 10100010 \\
\hline 11011000 & 00000000 & 00011000 & 00001000 & 10011000 & 00001000 & 01011000 & 10000000 \\
\hline 10101000 & 01001001 & 01101000 & 01001000 & 11101000 & 01001001 & 11101000 & 00001001 \\
\hline 10000000 & 00110010 & 10000000 & 10010000 & 10000000 & 00111010 & 00000000 & 10110010 \\
\hline 00111111 & 00101010 & 00001111 & 00111000 & 00101001 & 00111001 & 01000011 & 00110001 \\
\hline 00000100 & 00111010 & 00100100 & 00011010 & 00110010 & 00010010 & 10111110 & 00010100 \\
\hline 10001011 & 11011011 & 01011000 & 10010011 & 11011000 & 10010010 & 01010100 & 10010101 \\
\hline 10001100 & 01100001 & 00101001 & 11100100 & 01101001 & 11101100 & 01100101 & 11100101 \\
\hline 10110110 & 11100010 & 10110110 & 11000010 & 10110110 & 10010110 & 01000110 & 00000111 \\
\hline 11011000 & 00000010 & 11001000 & 00010000 & 00001000 & 00011000 & 01111000 & 01111000 \\
\hline 10101000 & 11101100 & 10001000 & 01101001 & 01001000 & 01101000 & 11001000 & 00001001 \\
\hline 10100000 & 00110000 & 10010000 & 00100010 & 10010000 & 10000000 & 10010000 & 10111010 \\
\hline 00011001 & 00100001 & 01011001 & 00100001 & 00110111 & 00001010 & 00011110 & 00001110 \\
\hline 00001101 & 00111100 & 10011011 & 00110100 & 00000100 & 00111010 & 00010010 & 00110010 \\
\hline 00010100 & 11010000 & 00010100 & 11010000 & 10000011 & 01001101 & 00000011 & 01001001 \\
\hline 10100101 & 01100111 & 11100101 & 01101111 & 10001100 & 01000001 & 11001100 & 01001001 \\
\hline 11000110 & 01000010 & 11000110 & 00010111 & 00010011 & 11100010 & 10010011 & 10110011 \\
\hline 01111000 & 01010000 & 00111000 & 01011000 & 11010000 & 00100010 & 10010000 & 10000000 \\
\hline 00001000 & 00011001 & 01001000 & 00011001 & 10101000 & 11001100 & 11101000 & 11001000 \\
\hline 11010000 & 10010010 & 11010000 & 10011010 & 10101000 & 00110000 & 00101000 & 00111000 \\
\hline 00111101 & 00001010 & 00101000 & 00011111 & & & & \\
\hline 00000001 & 00111010 & 00101011 & 00010000 & & & & \\
\hline 10001001 & 01000010 & 11001001 & 00000010 & & & & \\
\hline 10001001 & 01001110 & 00001001 & 11001110 & & & & \\
\hline 10110011 & 11010010 & 11110011 & 10010010 & & & & \\
\hline 10000000 & 00010010 & 00000000 & 10010010 & & & & \\
\hline 00001000 & 11111100 & 01001000 & 10111100 & & & & \\
\hline 11111000 & 00000000 & 01111000 & 10000000 & & & & \\
\hline
\end{tabular}

Table 2: Nonisomorphic pairs $B_{i}, B_{i}^{\prime}=R_{2}^{\top} B_{i} R_{2}(i=21, \ldots, 38)$ mentioned in Lemma 9. 
for which there exist an $\mathbb{R}$-cospectral mate (that is, the graph is not determined by the generalized spectrum); the column carries the name $A \& \bar{A}$. The table also presents the number of graphs for which a nonisomorphic cospectral mate can be obtained by GMswitching (the name of the column is GM). If $n \leqslant 8$, only GM-switching with respect to four vertices can give nonisomorphic mates. Therefore, nonisomorphic pairs related by GM-switching must be semi-isomorphic when $n \leqslant 8$. For $n \leqslant 6$, all graphs are determined by their generalized spectrum. On seven vertices, there exist 1044 graphs. Out of these, 40 graphs are not determined by the generalized spectrum, but for each of these graphs there exist a semi-isomorphic mate by GM-switching. Thus, every graph on seven vertices which is not determined by its generalized spectrum, is semi-isomorphic to some other graph. On eight vertices, there are 12346 nonisomorphic graphs. Out of these 1166 are not determined by their generalized spectrum, and for 1054 of these, an $\mathbb{R}$-cospectral mate can be obtained by GM-switching. Ted Spence (private communication) generated the remaining 112 graphs, and we compared these with the 427 graphs, for which six, seven or eight vertex switching applies. Only 44 of the 112 graphs in Spence's list did not occur in our list of 427 . These 44 graphs consist of 22 pairs of $\mathbb{R}$-cospectral graphs, which are not isomorphic or semi-isomorphic. Thus we have:

Proposition 10. On eight vertices, there exist 22 pairs of nonisomorphic $\mathbb{R}$-cospectral graphs for which no graph is semi-isomorphic with another graph. These are the twelve pairs of graphs displayed in Table 3, together with their complements (the last two pairs of the table are self-complementary).

According to Theorem 1, each of the 22 pairs of matrices from Proposition 10 are similar by a regular orthogonal matrix $Q$. For example for the first pair in Table 3 we find

$$
Q=\frac{1}{3}\left[\begin{array}{rrrrrrrr}
1 & 1 & 1 & 2 & -1 & -1 & 0 & 0 \\
1 & 1 & 1 & -1 & 2 & -1 & 0 & 0 \\
1 & 1 & 1 & -1 & -1 & 2 & 0 & 0 \\
2 & -1 & -1 & 1 & 1 & 1 & 0 & 0 \\
-1 & 2 & -1 & 1 & 1 & 1 & 0 & 0 \\
-1 & -1 & 2 & 1 & 1 & 1 & 0 & 0 \\
0 & 0 & 0 & 0 & 0 & 0 & 3 & 0 \\
0 & 0 & 0 & 0 & 0 & 0 & 0 & 3
\end{array}\right]
$$

which is a regular orthogonal matrix of level 3.

Warning. The 22 pairs of Proposition 10 are not the only $\mathbb{R}$-cospectral pairs that are not semi-isomorphic with each other. For example $\Gamma_{1}$ and $\Gamma_{2}$ from Example 4 have the same property, but the two graphs nor the complements do occur in Table 3. The reason is that both graphs have a nonisomorphic cospectral mate by GM-switching, therefore they are both semi-isomorphic with another graph, but not with each other.

Lemmas 8 and 9 show graphs which are semi-isomorphic by an indecomposable matrix $Q$. However, the computer investigations revealed that in all these cases there is also an decomposable $Q$ that establishes the semi-isomorphism. We know of no pair of graphs which are semi-isomorphic only by an indecomposable regular orthogonal matrix of level 2 . 


\begin{tabular}{|c|c|c|c|c|c|c|c|}
\hline 01110010 & 01110011 & 01111000 & 01111100 & 01111000 & 01111100 & 01111000 & 01111100 \\
\hline 10000011 & 10000010 & 10110000 & 10110000 & 10110100 & 10111000 & 10110100 & 10110010 \\
\hline 10000010 & 10000010 & 11000100 & 11001000 & 11001000 & 11000010 & 11001010 & 11001000 \\
\hline 10001101 & 10001100 & 11000100 & 11000011 & 11000000 & 11000000 & 11000001 & 11000001 \\
\hline 00010000 & 00010001 & 10000111 & 10100000 & 10100100 & 11000000 & 10100001 & 10100001 \\
\hline 00010000 & 00010000 & 00111010 & 10000011 & 01001011 & 10000011 & 01000001 & 10000010 \\
\hline 11100000 & 11100000 & 00001100 & 00010100 & 00000100 & 00100100 & 00100000 & 01000100 \\
\hline 01010000 & 10001000 & 00001000 & 00010100 & 00000100 & 00000100 & 00011100 & 00011000 \\
\hline 01111100 & 01111100 & 01111100 & 01111100 & 01111100 & 01111100 & 01111100 & 01111100 \\
\hline 10110010 & 10111000 & 10110010 & 10110010 & 10110010 & 10110010 & 10110010 & 10110010 \\
\hline 11000010 & 11010011 & 11001000 & 11001001 & 11001000 & 11001010 & 11001001 & 11001010 \\
\hline 11000010 & 11100000 & 11000001 & 11000100 & 11000001 & 11000001 & 11000100 & 11001001 \\
\hline 10000111 & 11000000 & 10100100 & 10100000 & 10100101 & 10100000 & 10100100 & 10110000 \\
\hline 10001000 & 10000011 & 10001011 & 10010001 & 10001000 & 10000000 & 10011010 & 10000000 \\
\hline 01111000 & 00100100 & 01000100 & 01000001 & 01000000 & 01100001 & 01000100 & 01100001 \\
\hline 00001000 & 00100100 & 00010100 & 00100110 & 00011000 & 00010010 & 00100000 & 00010010 \\
\hline 01111100 & 01111100 & 01111100 & 01111110 & 01111100 & 01111100 & 01111110 & 01111110 \\
\hline 10110010 & 10111010 & 10110011 & 10110001 & 10110010 & 10110010 & 10111001 & 10111110 \\
\hline 11001010 & 11000110 & 11001010 & 11001000 & 11001000 & 11001010 & 11010001 & 11011000 \\
\hline 11000011 & 11000100 & 11000000 & 11000100 & 11000011 & 11000101 & 11100001 & 11100000 \\
\hline 10100001 & 11000001 & 10100100 & 10100010 & 10100101 & 10100001 & 11000001 & 11100000 \\
\hline 10000000 & 10110001 & 10001001 & 10010000 & 10001010 & 10010000 & 10000000 & 11000001 \\
\hline 01110001 & 01100000 & 01100000 & 10001001 & 01010100 & 01100001 & 10000000 & 11000000 \\
\hline 00011010 & 00001100 & 01000100 & 01000010 & 00011000 & 00011010 & 01111000 & 00000100 \\
\hline
\end{tabular}

Table 3: Pairs of $\mathbb{R}$-cospectral graphs mentioned in Proposition 10

Acknowledgement. We thank Ted Spence for the 112 graphs on 8 vertices for which an $\mathbb{R}$-cospectral mate exists, but not by GM-switching.

\section{References}

[1] A.E. Brouwer, W.H. Haemers, Spectra of Graphs, Springer, 2012.

[2] H.C. Chan, C.A. Rodger, J. Seberry, On inequivalent weighing matrices, Ars combinatorica 21-A (1986), 299-333.

[3] C.R. Johnson, M. Newman, A note on cospectral Graphs, J. Combin. Theory Ser. B 28 (1980), 96-103.

[4] E.R. van Dam, W.H. Haemers, J.H. Koolen, Cospectral graphs and the generalized adjacency matrix, Linear Algebra Appl. 423 (2007), 33-41.

[5] C.D. Godsil, B.D. McKay, Constructing cospectral graphs, Aequationes Math. 25 (1982), 257-268.

[6] W.H. Haemers, E. Spence, Enumeration of cospectral graphs, Eur. J. Combinatorics 25 (2004), 199-211. 
[7] W. Wang, C.-X. Xu, An excluding algorithm for testing whether a family of graphs are determined by their generalized spectra, Linear Algebra Appl. 418 (2006), 62-74.

[8] W. Wang, C.-X. Xu, On the asymptotic behavior of graphs determined by their generalized spectra, Discrete Math. 310 (2010), 70-76. 